\title{
Unusual angiographic finding in acute coronary syndrome
}

\author{
Fernando Sabatel-Perez, Finn Akerström, Belen Santos Gonzalez, Luis Rodríguez-Padial \\ Department of Cardiology, Complejo Hospitalario de Toledo. Toledo, Spain
}

A 61-year-old woman without significant past medical history presented with acute chest pain and electrocardiographic signs of inferior wall ischemia as well as elevated troponin levels (Fig. 1A). The coronary angiogram showed absence of significant coronary artery disease, however, a nodular mass at the atrial level with its vascularization depending on the posterior descending artery could be observed (Fig. 1; Suppl. Video). The echocardiography (Fig. 1C) and computed tomography scan (Fig. 1D) revealed an intracardiac sessile mass $(43 \times 69 \times 33 \mathrm{~mm})$ localized to the left atrium and attached to the interatrial septum. One week later the patient underwent surgical excision of the tumor, which was of myxoid consistency (Fig. 1E), and histology confirmed a diagnosis of cardiac myxoma (Fig. 1F). Cardiac myxoma, the most prevalent primary cardiac tumor, commonly presents with embolic complications, although acute coronary syndrome is extremely rare with an incidence of $0.06 \%$. As in the present case, the right coronary artery is most frequently affected, although $20-60 \%$ of patients do not present any obstructive lesions at the time of coronary angiography, possibly explained by spontaneous solution of the embolus. An alternative pathophysiological hypothesis is a coronary steal phenomenon caused by the tumor neovascularization resulting in angina or acute coronary syndrome. The definitive treatment is surgical excision.

Conflict of interest: None declared

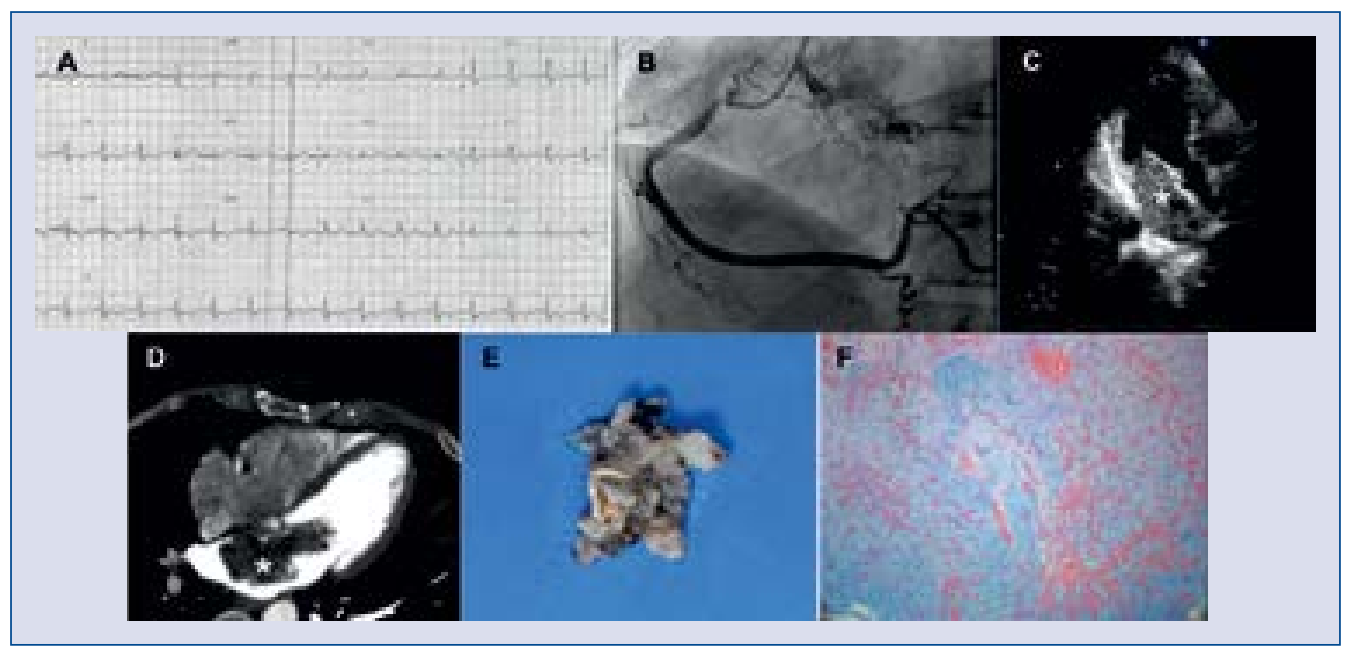

Figure 1. A. Electrocardiogram showing signs of acute inferior wall ischemia; B. Tumor neovascularization dependent on the posterior descending artery can be visualized during right coronary artery angiography; $\mathbf{C}$. Echocardiography and computed tomography (D) scan revealed a large intracardiac sessil mass $(43 \times 69 \times 33 \mathrm{~mm})$ localized to the left atrium and attached to the interatrial septum; E. The excised tumor was histologically confirmed to be of myxoid origin (hematoxylin-eosin and alcian blue stain) (F).

Address for correspondence: Dr. Finn Akerström, $\mathrm{MBChB}$, Cardiology Department, Hospital Virgen de la Salud, Avda. Barber 30, Planta Semisótano, 45004, Toledo, Spain, e-mail: finnakerstrom@gmail.com

Received: 24.05.2018 Accepted: 12.06.2018 